\title{
Targeting the MAP kinase pathway in astrocytoma cells using a recombinant anthrax lethal toxin as a way to inhibit cell motility and invasion
}

\author{
SALEH AL-DIMASSI ${ }^{1 *}$, GILBERT SALLOUM ${ }^{1 *}$, BECHARA SAYKALI $^{1}$, OULA KHOURY $^{1}$, \\ SHIHUI LIU ${ }^{2}$, STEPHEN H. LEPPLA ${ }^{2}$, RALPH ABI-HABIB ${ }^{1}$ and MIRVAT EL-SIBAI ${ }^{1}$ \\ ${ }^{1}$ Department of Natural Sciences, The Lebanese American University, Beirut 1102 2801, Lebanon; \\ ${ }^{2}$ Microbial Pathogenesis Section, Laboratory of Parasitic Diseases, National Institutes of Health, Bethesda, MD 20892, USA
}

Received September 28, 2015; Accepted November 5, 2015

DOI: 10.3892/ijo.2016.3431

\begin{abstract}
Malignant astrocytomas are highly invasive into adjacent and distant regions of the normal brain. Understanding and targeting cancer cell invasion is an important therapeutic approach. Cell invasion is a complex process that replies on many signaling pathways including the mitogen-activated protein (MAP) kinase (MAPK). In many cell lines, the use of MAPK-targeted drugs proved to be a potential method to inhibit cancer cell motility. In the present study, we use a recombinant anthrax lethal toxin (LeTx), which selectively inhibits the MAPK pathway, in order to target invasion. LeTx proved ineffective on cell survival in astrocytoma (as well as normal cells). However, astrocytoma cells that were treated with LeTx showed a significant decrease in cell motility as seen by wound healing as well as random 2D motility in serum. The cells also showed a decrease in invasion across a collagen matrix. The effect of LeTx on cell migration was mediated though the deregulation of Rho GTPases, which play a role in cell motility. Finally, the effect of LeTx on cell migration and Rho GTPases was mimicked by the inhibition of the MAPK pathway. In this study, we describe for the first time the effect of the LeTx on cancer cell motility and invasion not cell survival making it a potentially selective brain tumor invasion inhibitor.
\end{abstract}

\section{Introduction}

Approximately 40,000 brain tumor cases were reported between 1973 and 2001 (1). Astrocytomas, known as grade II

Correspondence to: Dr Mirvat El-Sibai, Department of Natural Sciences, Lebanese American University, P.O. Box 13-5053, Chouran, Beirut 1102 2801, Lebanon

E-mail: mirvat.elsibai@lau.edu.lb

${ }^{*}$ Contributed equally

Key words: anthrax toxin, astrocytomas, motility, invasion, MAPK, RhoA astrocytic tumors (2), have a peak incidence in early adulthood, and are the most common form of primary human brain tumors that are usually associated with a poor prognosis (3). Systemic metastasis of malignant astrocytomas rarely occurs. These tumors; however, are highly invasive into adjacent and distant tissues of the normal brain, which makes them surgically and medically unmanageable (4). Tumor invasion and metastasis are direct consequences of uncontrolled cell migration (5). The key to understanding these phenomena is to uncover altered cellular signaling underlying tumor aggressiveness.

Motility is a physiological process needed for immune responses, embryonic development and wound healing. Cancer cells use this process to invade neighboring tissues and to metastasize to distant organs ensuring cancer progression (6). Cell motility is a complicated process that is tightly coordinated by the cell itself with the help of various proteins (7). Rho GTPases including RhoA, Rac and Cdc42 are considered key players and chief regulators of cell migration, among many other previously described cellular events $(8,9)$. These small monomeric $\mathrm{G}$ proteins act as molecular switches, their spatiotemporal regulation being tightly regulated (10). Upstream regulators include GTPase-activating proteins (GAPs), which catalyze the conversion of the active GTP-bound Rho-family GTPases to the inactive GDP-bound form, and Guaninenucleotide exchange factors (GEFs) that activate Rho GTPases by catalyzing the exchange of bound GDP to GTP (11). Upon stimulation with growth factors, intracellular Rho family small GTPases substitute their bound GDP to GTP. Once active, they interact with and activate specific downstream effectors mediating a multitude of intracellular responses including the reorganization of the actin cytoskeleton leading to cell migration and invasion (12-14). Altered levels of expression/activation of Rho GTPases have been implicated in the invasion and progression of many cancers (15-17). A previous study conducted in our laboratory showed that overexpression or knockdown of RhoA leads to the decrease in 2D motility though altered focal complex formation and maturation into focal adhesions. The same study concluded that RhoA undergoes an activation/inactivation cycle at the cell edges mediating protrusion formation (18). This mechanism plays a 
central role in tumor progression resulting in cancer spreading and invasion (19).

The MAPK cascade is an important signaling pathway that conveys extracellular signals into the nucleus. It is activated by various extracellular stimuli contributing to the regulation of cellular responses such as apoptosis, survival, proliferation and differentiation (20). After detecting an extracellular mitogen, Ras, a small GTPase, switches its GDP to GTP. This allows a subsequent activation of MAP3K (Raf), MAP2K (MEK) and MAPK (ERK1/2) respectively, activating downstream transcription factors (21). ERK/MAPK pathway seems to play an elaborate role in tumor progression since its inhibition abolishes the growth of BRAF-mutated HT29 colon cancer cells in mice (22). In ER-negative MDA-MB-468 and MDA-MB-231 cells, TGF $\alpha$-induced MAPK activation was correlated to an $\sim 5$-fold increase in cellular motility. Similar results were observed in ER-positive TGF $\alpha$-stimulated MCF-7 breast cancer cells with an $\sim 2$-fold increase in cell motility (23).

The relationship between ERK-MAPK and Rho GTPases in cell motility is far from being uncovered. Studies conducted on LN-18 cells revealed a gradual decrease in MAPK activation the longer the cells are treated with ROCK-inhibitor Y27632 (24). A recent study described two signaling pathways downstream from MAPK that regulate cell motility in colon carcinoma cells. Fra-1, member of theFos family, was shown to downregulate RhoA activity by inactivating $\beta 1$-integrin. The former step being necessary for urokinase-type plasminogen activator receptor (UPAR)-dependent activation of Rac, downstream MAPK-ERK, resulting in lamellipodial formation (21).

Anthrax lethal toxin (LeTx) is an exotoxin produced by the gram-positive bacterium Bacillus Anthasis. LeTx is a binary toxin that consists of two distinct proteins, the catalytic lethal factor (LF) and the cell binding and internalization protective antigen $(\operatorname{PrAg})(25,26)$. These two protein subunits act together to impart their physiological effects. PrAg binds to cells though its cell surface receptors capillary morphogenesis gene-2 (CMG2) and tumor endothelial marker-8 (TEM8) and is cleaved by furin-like proteases releasing a $20-\mathrm{kDa}$ fragment and generating $\operatorname{PrAg}_{63}$, a 63-kDa active fragment $(27,28)$. Three or four LF molecules bound to $\operatorname{PrAg}_{63}$ undergo endocytosis. The latter, upon endosome acidification, undergoes a conformational change leading to the formation of pores allowing LF translocation into the cytosol (29). Once in the cytosol, the enzymatic subunit LF disrupts various cell processes notably cellular signaling. Previous studies revealed that the LF/PA mixture significantly impaired chemotaxis among polymorphonuclear neutrophils (PMNs) and abolished cellular polarity $(30,31)$. Upon screening for novel inhibitors of MAPK signal transduction pathways, activity profile of LeTx was similar to PD09859, a selective inhibitor of the MAPK pathway (32). Later on, lethal factor of LeTx (LF) was identified as a endoprotease that inhibits the MAPK pathway by cleaving the amino terminus and inactivating MEKs subsequently leading to growth inhibition and death $(33,34)$.

The aim of the present study was to investigate the effect of LeTx on motility and invasion of astrocytomas cancer cell lines. First, we studied its effect on cellular proliferation and viability upon treating cells with recombinant toxin. Then, we examined its effect on 2D motility, adhesion and invasion of astrocytoma cells, in addition to identify its effect on Rho
GTPases. Our results revealed MAPK-mediated inhibition of random 2D-motility and 3D-motility in collagen. Decrease in the motile and invasive profile of astrocytoma cells was opposed to an increase in RhoA activity previously shown to cause cell stabilization that contradicts free invasion. LeTx did not seem have an effect on the activation of PI3K, a signal transduction pathway involved in cell survival and proliferation.

\section{Materials and methods}

Cell culture. Human astrocytoma cell line SF268, obtained from Dr Marc Symons, was cultured in Dulbecco's modified Eagle's medium (DMEM) supplemented with $10 \%$ fetal bovine serum (FBS) and $100 \mathrm{U}$ penicillin/streptomycin at $37^{\circ} \mathrm{C}$ and $5 \% \mathrm{CO}_{2}$ in a humidified chamber.

Proliferation inhibition assay (cytotoxicity). Sensitivity of astrocytoma cell line to LeTx (PrAg/LF) was determined using a proliferation inhibition assay as previously described. Briefly, aliquots of 1,000 cells/well, in $100 \mu \mathrm{l}$ of cell culture medium, containing a fixed concentration of $10^{-9} \mathrm{M} \mathrm{LF}$, were plated onto a flat-bottom 96-well plate (Corning Inc., Corning, NY, USA). Then, $50 \mu \mathrm{l}$ of PrAg in media were added to each well to yield concentrations ranging from $10^{-8}$ to $10^{-13} \mathrm{M}$. Following a 72 -h incubation at $37^{\circ} \mathrm{C} / 5 \% \mathrm{CO}_{2}, 50 \mu \mathrm{l}$ of XTT cell proliferation reagent (Roche, Basel, Switzerland) was added to each well and the plates incubated for another $4 \mathrm{~h}$. Absorbance was then read at $450 \mathrm{~nm}$ using a microplate reader (Thermo Fisher Scientific, Waltham, MA, USA). Absorbance was plotted against the log of concentration, and a non-linear regression with a variable slope sigmoidal dose-response curve was generated along with inhibitory concentration 50 $\left(\mathrm{IC}_{50}\right)$ using GraphPad Prism 5 software (Graphpad Software Inc., San Diego, CA, USA).

Antibodies and reagents. Mouse monoclonal anti-ERK antibody, mouse monoclonal anti-vinculin antibody, rabbit polyclonal anti-PERK antibody was obtained from Santa Cruz Biotechnology (Santa Cruz, CA, USA). Mouse monoclonal anti-RhoA, rabbit polyclonal antibody to pan-AKT, rabbit polyclonal antibody to pan-AKT (phospho T308) from Abcam.

Fluorescent secondary antibodies (Alexa Fluor 488) were obtained from Invitrogen. To visualize the actin cytoskeleton, cells were stained with rhodamin phalloidin (Invitrogen).

Treatment with toxin. Sublethal anthrax lethal toxin concentration range was determined after a literature review. A $2.84 \mathrm{mg} / \mathrm{ml}$ lethal factor (LF) and a $5.23 \mathrm{mg} / \mathrm{ml}$ protective antigen (PA) were diluted separately in DMEM to reach a concentration ratio of $1: 3$, respectively. Cells were treated by adding equal volumes of each of the two dilutions for $2 \mathrm{~h}$. Following treatment cells were left for $24 \mathrm{~h}$ in complete medium before running the desired experiment.

Wound healing assay. Cells were grown to confluence on culture plates and a wound was made in the monolayer with a sterile pipette tip. Cells were then washed twice with PBS to remove debris and new medium was added. Phase-contrast images of the wounded area were captured at 0 and $24 \mathrm{~h}$ after 
wounding. Wound widths were measured at 11 different points for each wound, and the average rate of wound closure was calculated (in $\mu \mathrm{m} / \mathrm{h}$ ).

Motility assay/analyzing 2D motility. For motility analysis, images of cells moving randomly in serum were collected every $60 \mathrm{sec}$ for $2 \mathrm{~h}$ using a 20x objective. During imaging, the temperature was controlled using a Nikon heating stage which was set at $37^{\circ} \mathrm{C}$. The medium was buffered using HEPES and overlayed with mineral oil. The speed of cell movement was quantified using the ROI tracker plugin in ImageJ software, which was used to calculate the total distance travelled by individual cells. The speed is then calculated by dividing this distance by the time (120 $\mathrm{min})$ and reported in $\mu \mathrm{m} / \mathrm{min}$. The speed of at least 15 cells for each condition was calculated. The net distance travelled by the cell was calculated by measuring the distance travelled between the first and the last frames.

Adhesion assay. Plates (96-well) were coated with collagen using collagen solution, type I from rat tail (Sigma) overnight at $37^{\circ} \mathrm{C}$ then washed with washing buffer (0.1\% BSA in DMEM). The plates were then blocked with $0.5 \%$ BSA in DMEM at $37^{\circ} \mathrm{C}$ in a $\mathrm{CO}_{2}$ incubator for $1 \mathrm{~h}$. Then washing the plates and chilling them on ice followed. The cells were trypsinized and counted to $4 \times 10^{5} \mathrm{cell} / \mathrm{ml}$. A total of $50 \mathrm{ml}$ of cells were added in each well and incubated at $37^{\circ} \mathrm{C}$ in a $\mathrm{CO}_{2}$ incubator for $30 \mathrm{~min}$. The plates were then shaken and washed 3 times. Cells were then fixed with $4 \%$ paraformaldehyde at room temperature for $10 \mathrm{~min}$, washed, and stained with crystal violet $(5 \mathrm{mg} / \mathrm{ml}$ in $2 \%$ ethanol) for $10 \mathrm{~min}$. Following the staining with crystal violet, the plates were washed extensively with water, and left to dry completely. Crystal violet was solubilized by incubating the cells with $2 \%$ SDS for $30 \mathrm{~min}$. The absorption of the plates was read at $550 \mathrm{~mm}$ using a plate reader.

Immunostaining. The cells were plated on coverslips, and the appropriate treatment was applied. Cells were fixed with $4 \%$ paraformaldehyde for $10 \mathrm{~min}$, and permeabilized with $0.5 \%$ Triton X-100 for $10 \mathrm{~min}$. To decrease background fluorescence, cells were rinsed with $0.1 \mathrm{M}$ glycine then incubated with $0.1 \mathrm{M}$ glycine for $10 \mathrm{~min}$. For blocking, cells were incubated 4 times with $1 \%$ BSA, $1 \%$ FBS in PBS for 5 min. Samples were stained with primary antibodies for $2 \mathrm{~h}$ and with fluorophoreconjugated secondary antibodies for $2 \mathrm{~h}$. Fluorescent images were taken using a x60 objective on a fluorescent microscope.

Boyden chamber/invasion assay. Cells were treated with toxin or left untreated as control, and the invasion assay was performed following the treatment period using the collagen-based invasion assay (Millipore) according to the manufacturer's instructions. Briefly, $24 \mathrm{~h}$ prior to the assay, cells were starved with serum-free medium. Cells were harvested, centrifuged and then resuspended in quenching medium (without serum). Cells were then brought to a concentration of $1 \times 10^{6}$ cells $/ \mathrm{ml}$. In the meantime, inserts were prewarmed with $300 \mu \mathrm{l}$ of serum-free medium for $30 \mathrm{~min}$ at room temperature. After rehydration, $250 \mu \mathrm{l}$ of medium was removed from the inserts, and $250 \mu \mathrm{l}$ of cell suspension was added. Inserts were then placed in a 24 -well plate, and $500 \mu \mathrm{l}$ of complete medium (with $10 \%$ serum) was added to the lower wells. Plates were incubated for $24 \mathrm{~h}$ at $37^{\circ} \mathrm{C}$ in a $\mathrm{CO}_{2}$ incubator. Following the incubation period, inserts were stained for $20 \mathrm{~min}$ at room temperature with $400 \mu \mathrm{l}$ of cell stain provided with the kit. The stain was then extracted with extraction buffer (also provided). The extracted stain $(100 \mu \mathrm{l})$ was then transferred to a 96-well plate suitable for colorimetric measurement using a plate reader. Optical density was then measured at $560 \mathrm{~nm}$.

Western blotting. Cell lysates were prepared by scraping the cells in a sample buffer (4\% SDS, $10 \% \beta$-mercaptoethanol, $20 \%$ glycerol, $0.004 \%$ bromophenol blue, and $0.125 \mathrm{M}$ Tris- $\mathrm{HCl}$ at a $\mathrm{pH}$ of 6.8). The resulting lysates were boiled for 5 min. Protein samples were separated by SDS-PAGE on $10 \%$ (for ERK and P-ERK) or 15\% (for RhoA) gels and transferred to PVDF membranes overnight at $30 \mathrm{~V}$. The membranes were then blocked with 5\% non-fat dry milk in PBS containing $0.1 \%$ Tween-20 for $1 \mathrm{~h}$ at room temperature and incubated with primary antibody at a concentration of $1: 100$ for $2 \mathrm{~h}$ at room temperature. After the incubation with the primary antibody, the membranes were washed and incubated with secondary antibody at a concentration of 1:1,000 for $1 \mathrm{~h}$ at room temperature. The membranes were then washed, and the bands visualized by treating the membranes with western blotting chemiluminescent reagent ECL (GE Healthcare). The results were obtained on X-ray film (Agfa Healthcare).

Pull-down assay. Cells were lysed and incubated with GST-RBD or GST-CRIB and the pull-down assay was performed using the RhoA/Rac1/Cdc42 Activation Assay Combo kit (Cell Biolabs Inc., San Diego, CA, USA) following the manufacturer's instructions. Lysates were incubated with GST-RBD (for RhoA) or GST-CRIB for Rac for $1 \mathrm{~h}$ at $4^{\circ} \mathrm{C}$. GTP-RhoA and GTP-Rac were detected by western blotting using the anti-RhoA and the anti-Rac antibodies provided in the kit. Total proteins were collected prior to the incubation with GST beads and used as a loading control.

\section{Results}

LeTx acts though the MAPK pathway. LeTx is known to inhibit the MAPK pathway in part by inducing the cleavage of MEK upstream of ERK $(20,21)$. Western blots revealed a decrease in the activation of ERK as seen in the decrease in p-ERK levels compared to total ERK levels upon LeTx or U0126 treatment as expected (Fig. 1A). Paradoxically, LeTx has no net effect on astrocytoma cell viability. Using an increasing concentration of recombinant anthrax lethal toxin followed by the evaluation of cell viability via XTT, revealed no significant effect of the toxin on astrocytoma cells viability (Fig. 1B). Cells are slightly sensitive to $\mathrm{PrAg} / \mathrm{LF}$, indicating a partial resistance to the LF-mediated inhibition of the MAPK pathway.

LeTx decreases astrocytoma invasion. Since LeTx had no effect on astrocytoma viability, we wanted to test its potential effect on astrocytoma cell invasion. In order to do so, we performed an in vitro Transwell migration assay using FBS as a chemoattractant. A negative control was run in parallel whereby serum-free media was introduced into the well and the corresponding insert. The results showed a 2 -fold decrease 
$\mathbf{A}$

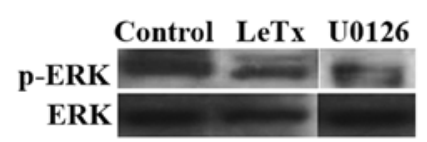

B

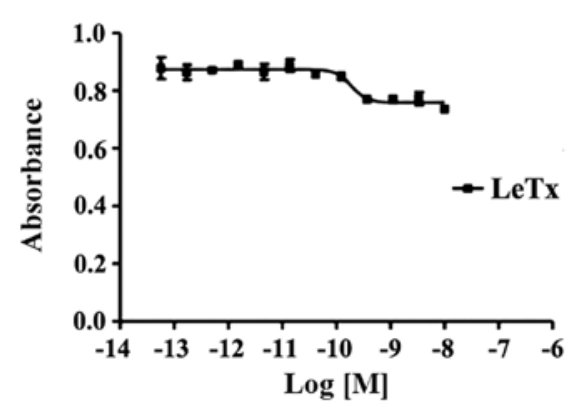

Figure 1. Recombinant anthrax lethal toxin inhibits the MAPK pathway without affecting cell viability in astrocytoma. (A) SF268 cells were treated either with the recombinant anthrax lethal toxin or the MEK inhibitor U0126 for $2 \mathrm{~h}$. Cells were lysed and immunoblotted by western blot analysis for p-ERK (upper gel) or ERK (lower gel) for loading control. Quantitation represents three different blots from three independent experiments. (B) The effect of LeTx on cell viability was tested using an MTT assay as mentioned in Materials and methods. The graph is a non-linear regression curve of LeTx on human SF-268 cell lines.

in cellular invasion in treated cells as compared to control (Fig. 2).

LeTx decreases astrocytoma cell motility. In order to further study the effect of LeTx on astrocytoma invasion we looked at the behavior of the cells in 2D in order to observe their phenotype. First the $2 \mathrm{D}$ migration was examined by performing a wound closure assay. Treatment with LeTx caused decrease in the rate of wound closure from 11 to $4 \mu \mathrm{m} / \mathrm{h}$ (Fig. 3A and B). The area of the wounds we calculated both at time 0 and $24 \mathrm{~h}$ after inflicting the wound (Fig. 3B). The results reveal that control cells were able to close $>50 \%$ of the wound after $24 \mathrm{~h}$, as opposed to treated cells where only $20 \%$ of the wound was closed (Fig. 3B). The net path taken by individual cells significantly decreased $>2.5$-fold in cells treated with LeTx or with the MEK $1 / 2$ inhibitor U0126 as determined by time-lapse imaging to detect random 2D cell migration rates and profiles (Fig. 3D). Average speed of individual cells also significantly decreased upon treatment from $\sim 0.45$ to $\sim 0.2 \mu \mathrm{m} / \mathrm{min}$ (Fig. 3D). Time lapse movies allowed us to examine the migration profile and the phenotype of individual cells in response to treatment with LeTx. Treated cells displayed an extended shape with thin elongated protrusions. Cells treated with LeTx seemed to lack de-adhesion and to be unable to retract their tail at times (Fig. 3C) which might explain the decrease in cell migration. This phenotype was not, however, seen in cells treated with U0126 which suggest another mechanism through which this drug is affecting migration in these cells.

LeTx increases cell adhesion. Having suspected an inability of the cells to detach during migration in the previous experiment which might indicate exaggerated adhesions, we were interested to look at the effect of LeTx on cell adhesion. Cells treated with LeTx displayed a 2-fold increase in cellular adhesion as compared to control cells (Fig. 4A) which is consistent with the elongated phenotype. The elongated phenotype was also reminiscent of a phenotype we have previously reported in astrocytoma cells where the RhoA GAP StarD13 was knocked down which leads to an increase in RhoA activity and adhesion structures resulting in the inability of cells to migrate (18).

Indeed, cells treated with LeTx treated cells exhibited a higher density of intracellular stress fibers as reflected with phalloidin staining which might indicate an increase in RhoA activity. In addition, immunostaining the cells with antivinculin antibody revealed focal adhesions that were more prevalent in treated cells. Large punctate structures were
A

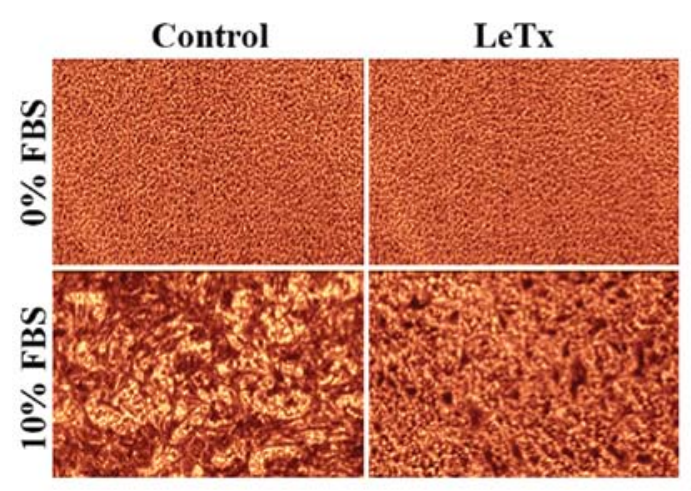

B

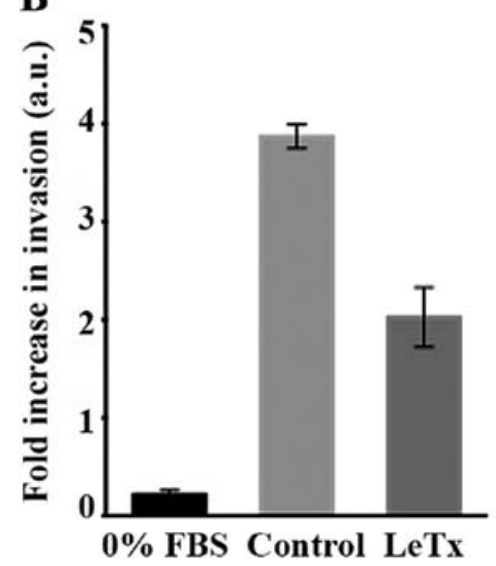

Figure 2. Recombinant anthrax lethal toxin decreases cellular invasion. (A) Representative micrographs of invaded cells on the bottom side of the collagencoated membrane stained with cell stain according to assay instructions. SF268 treated with the toxin and control cells were allowed to invade towards $10 \%$ FBS for $24 \mathrm{~h}$. Cells $/ \mathrm{ml}\left(1 \times 10^{6}\right)$ were used in each assay. (B) Quantitation of (A) whereby the cell stain was solubilized using extraction buffer and absorption of cells suspensions were measured at $550 \mathrm{~nm}$. Data are reported in arbitrary units and normalized to the control. Data are the mean \pm SEM from three experiments. 
A
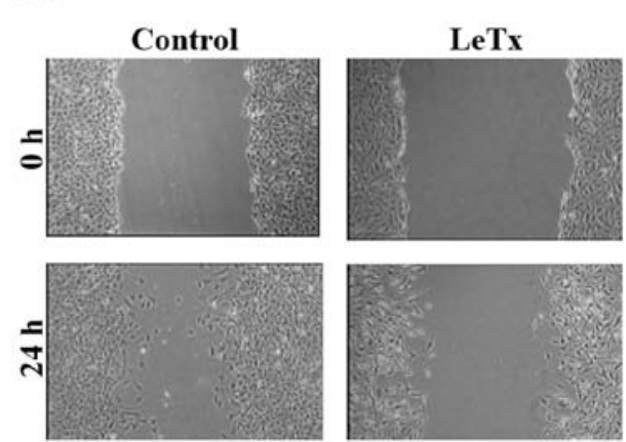

B

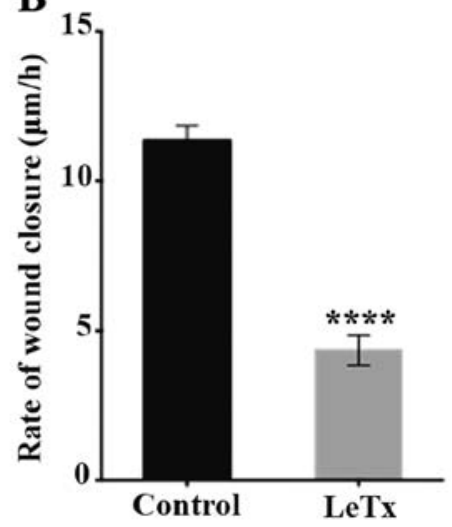

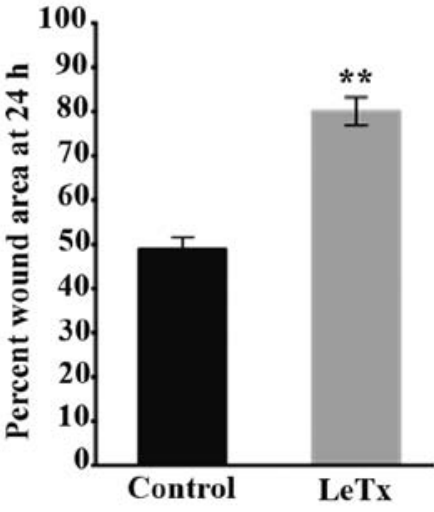

C
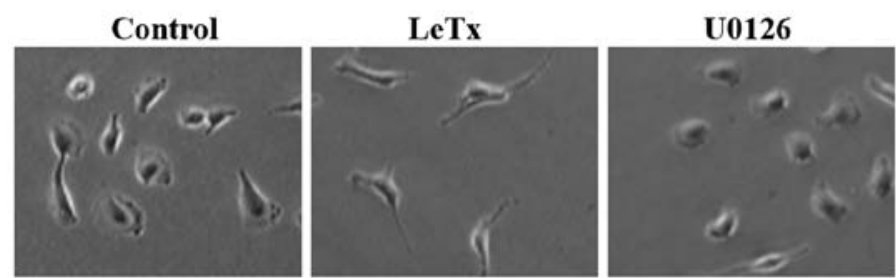

D
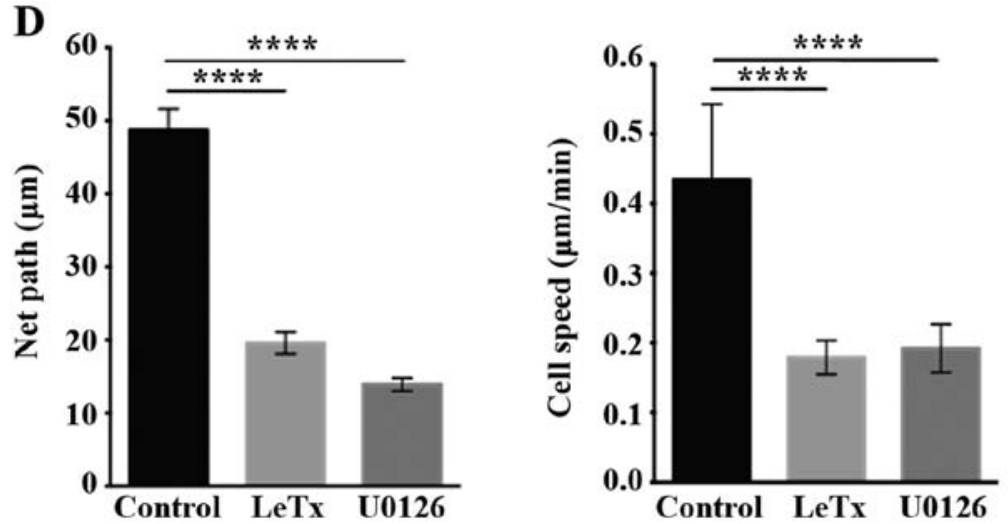

Figure 3. Recombinant anthrax lethal toxin inhibits 2D motility. (A) Cells treated with the recombinant anthrax lethal toxin for $2 \mathrm{~h}$ were grown in a monolayer then wounded and left to recover the wound then imaged at the same frame after $24 \mathrm{~h}$ (lower micrographs). Scale bar, $50 \mu \mathrm{m}$. (B) Quantitation for (A). Left panel, wound widths were measured at 12 different points for each wound, and the average rate of wound closure for the cells was calculated in $\mu \mathrm{m} / \mathrm{h}$. Right panel, percentages of wound area at $24 \mathrm{~h}$ following wounding. Data are the mean \pm SEM from three wounds closure assays from three independent experiments. ${ }^{* * * *} \mathrm{P}<0.0001$ indicates a statistically significant difference; ${ }^{* *} \mathrm{P}<0.002$ indicates statistically significant differences. (C) Representative micrographs of cells treated with either recombinant anthrax lethal toxin or the MEK inhibitor U0126 and allowed to undergo random motility in serum. Scale bar, $50 \mu \mathrm{m}$. (D) The net paths (left panel) or the cell speed of projected 120 frames from $2 \mathrm{~h}$ long time lapse movies were quantitated and expressed in $\mu \mathrm{m}$ and $\mu \mathrm{m} / \mathrm{min}$, respectively. Data are the mean \pm SEM from 15 movies. ${ }^{* * * *} \mathrm{P}<0.0001$ indicates statistically significant differences.

present at the leading edge and the tail as well as the cell body, unlike control cells where focal adhesions were mostly limited to the lamellipodium (Fig. 4B).

Effect of LeTx and U0126 on RhoA and Rac activation. It is well established in the literature that RhoA is a major contributor to the formation and maturation of focal complexes into focal adhesions. After being shown to increase cell adhesion, we aimed to check for the effect of LeTx on the activation of RhoA. Treated cells revealed a higher RhoA activity as compare to control (Fig. 5A). Treating the cells with U0126, however, did not lead to an increase in RhoA activation. This is in accordance with the phenotype seen in Fig. 3C where the cells treated with U0126 lacked the elongated phenotype seen in the cells treated with LeTx.
We also looked at the activation of Rac which is a major regulator of cell migration. In response to both LeTx and U0126 treatment, the activation of Rac was substantially decreased.

LeTx and U0126 treatments do not affect the PI3K pathway. Finally, since PI3K is a known activator or Rho GTPases (9), we examined whether LeTx and U0126 are exerting their effects on Rac or RhoA through affecting the PI3K pathway. Treating the cells with LeTx or U0126 did not affect the level of phosphorylation of Akt downstream of PI3K (Fig. 6).

\section{Discussion}

Invasiveness of brain carcinoma and its infiltration to the neighboring locations in brain limits the survival of patients 
$\mathbf{A}$
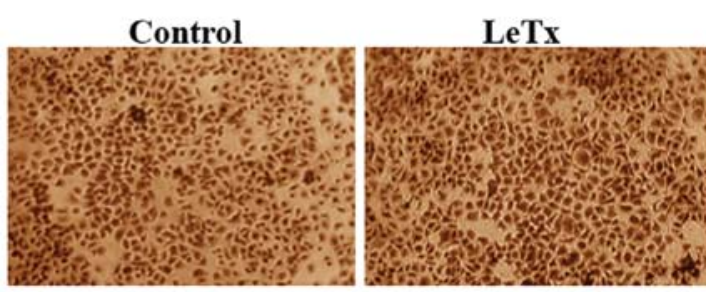

B

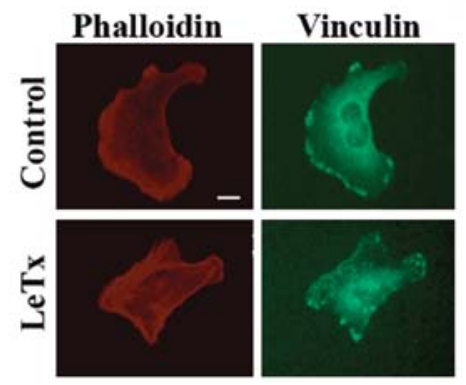

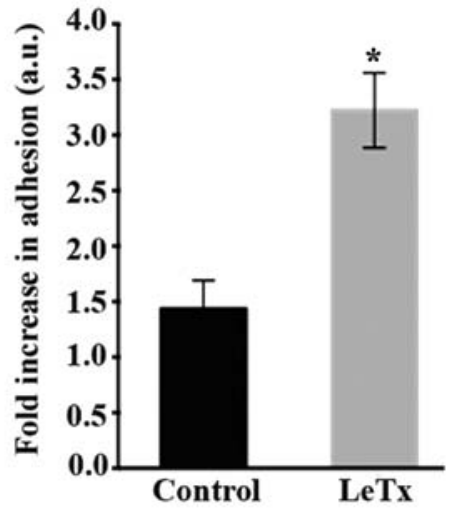

Figure 4. Recombinant anthrax lethal toxin increases cell adhesion. (A) Representative micrographs of cells fixed and stained with crystal violet to assess adhesion. Scale bar, $50 \mu \mathrm{m}$. The graph is a quantitation whereby crystal violet was solubilized and the absorption of the plates was read at $550 \mathrm{~nm}$ using an ELISA plate reader. Data are reported in arbitrary units and normalized to the control. Data are the mean \pm SEM from three experiments (15 cells/condition/ experiment). "P<0.01 indicates a statistically significant difference. (B) Representative micrographs of control cells and cells treated with the recombinant anthrax lethal toxin that were fixed and immunostained with vinculin. Scale bar, $10 \mu \mathrm{m}$.

$\mathbf{A}$

Control LeTx U0126

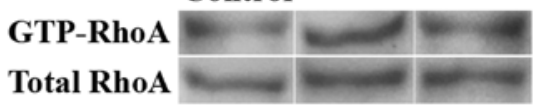

GTP-Rac

Total Rac

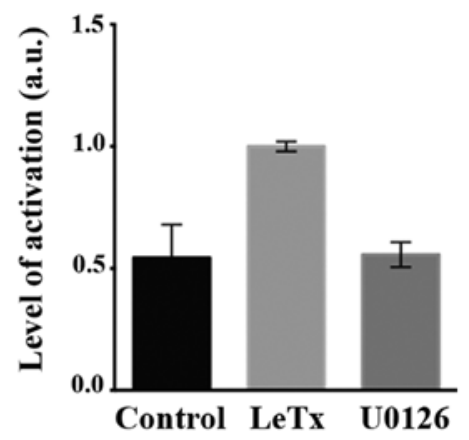

B

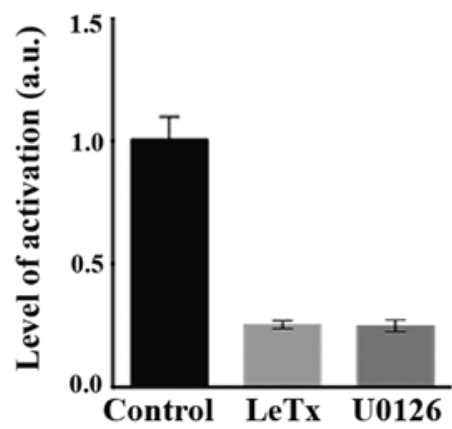

Figure 5. Effect of recombinant anthrax lethal toxin on RhoA and Rac activations. (A) SF268 cells were treated with LeTx or with U0261. The cells were then lysed and incubated with GST-RBD (Rhoteckin binding domain) to pull down active Rho. The samples were then blotted with Rho antibodies. The lower gel is a western blot analysis for the total cell lysates for loading control. The bands from the active RhoA gel were quantitated in the right panel using ImageJ and normalized to the amount of total proteins. Data are the mean \pm SEM of three blots from three independent experiments. (B) SF268 cells were treated as in A. The cells were then lysed and incubated with GST-CRIB to pull down active Rac. The samples were then blotted with Rac antibodies. The lower gel is a western blot analysis for the total cell lysates for loading control. The bands from the active Rac gel were quantitated in the right panel using ImageJ and normalized to the amount of total proteins. Data are the mean \pm SEM of three blots from three independent experiments.

from several years to several months, depending on the stage of this cancer. Previous studies were able to determine that inactivation of MAPK pathway though proteolysis was enough to kill different tumor types (35). A study by Huang et al (30) 

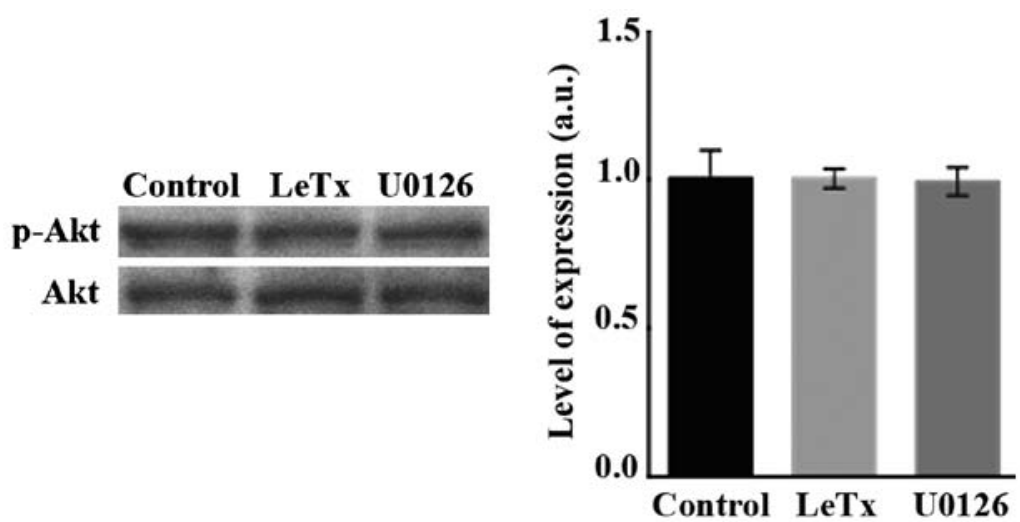

Figure 6. Recombinant anthrax lethal toxin effect on the PI3K pathway. SF268 cells were treated either with the recombinant anthrax lethal toxin or U0126 for $2 \mathrm{~h}$. Cells were lysed and immunoblotted by western blot analysis for p-Akt (upper gel) or Akt (lower gel) for loading control. Quantitation represents three different blots from independent experiments.

showed that renal cell carcinoma having high expression levels of MKK1 and ERK2 were sensitive to the fusion anthrax lethal toxin due to its capability of inhibiting the MAPK pathway leading to cell death.

Based on the ability of toxin to inhibit MAPK pathway resulting in cell death, astrocytoma cells were tested with an increasing gradient of toxin concentration to assess their sensitivity to the toxin. Surprisingly, no significant cytotoxic effect of toxin on astrocytoma cells was observed. A previous study aiming to unveil the mechanism of action of LeTx and its pathological/physiological effect on peripheral polymorphonuclear neutrophils (PMNs) showed that treating those cells with low concentrations of LeTx caused paralysis of directed migration and impaired chemokinesis and cell polarity, which was not accompanied by significant apoptosis or necrosis (31). This former study was among the few tackling the potential relationship between LeTx and impaired cellular movement. Additionally, most of the studies were conducted on immune cells and none on tumor cells. Hence, the significance of the present study as a novel approach to treat highly motile and invasive tumor cells with an agent that does not necessarily affect cell viability.

In the present study, treating astrocytoma cells with the toxin led to a decrease in 2D motility as shown by time-lapse movies and their quantitation in addition to wound-healing assay. It is important to note that upon treating the cells with toxin a change in cellular phenotype was observed where the cells gained an elongated morphology. Cells displayed an unusual shape with the absence of defined leading edge and tail. Over and above that, no matter how far the cell body migrated, the 'tail region' seemed to be stuck in place unable to detach and retract. This phenotype was previously observed in astrocytoma cells undergoing random 2D migration in serum whenever RhoA was overexpressed or a RhoA GAP called StarD13 was knocked down (18). We hypothesized that LeTx could be impairing cell migration, in part, through the deregulation of RhoA activation which leads to the phenotype observed.

As we have previously established (18), the activation of RhoA needs to cycle during the cell motility cycle. Initially, RhoA activation increases which is needed for focal adhesions to form which enables the protrusion to exert pressure on the
ECM to pull the cell forward. Following that, RhoA activation needs to decrease in order for the focal adhesion to dissolve for the cell tail to detach and for the cell to move forward. A persistent activation of RhoA was shown to inhibit cell migration in astrocytoma through persistent cell adhesions that disable the cells from moving. Consistently, in the present study when we treated the cells with LeTx, we observed an increase in cell adhesion and an exaggerated phenotype of focal adhesions and an increase in stress fiber formation which is indicative of an increase in RhoA activation.

For the above reason, a pulldown assay using Rhotekin RBD agarose beads was performed to pull the active RhoA. The results revealed an increase in the activation of RhoA suggesting that LeTx could be acting on RhoA to induce the changes observed in cell migration and invasion. The pulldown results also explain the changes in cellular morphology observed though time-lapse imaging whereby cells seemed to exhibit longer cellular extensions. This is due to the high RhoA activity causing stabilization of focal adhesions at the leading edge and the tail preventing forward protrusion and tail retraction. High RhoA activity also explains the remarkable presence of stress fibers observed upon staining for actin.

Our findings come in parallel with a decrease in MAPK activity upon toxin treatment, as compared to control and treatment using MAPK inhibitor U0126. Moreover, LeTx seems to have no effect on the activation of phosphatidylinositol 3-kinase (PI3K). This is in accordance with previously published works depicting the PI3K LeTx-resistance and the critical role of the PI3K/Akt/glycogen synthase kinase-3 $\beta$ signaling pathway in the protection and the recovery from LeTx-induced MEK cleavage in macrophages (36). Given its effect on RhoA, collectively this suggests that LeTx is not exerting its effect on RhoA through PI3K.

U0126 is a specific inhibitor of the MEK1/2 pathway whereas LeTx has a broader effect. Both agents led to a complete inhibition of Rac which is one of the main effectors of cell migration $(9,18,37)$. This would suggest that LeTx leads to the inhibition of cell migration and invasion, at least in part, through its inhibition of MEK1/2 and Rac downstream. However, as shown the MEK1/2 had no effect on RhoA and did not mimic the elongated phenotype seen in the LeTxtreated cells. This indicates that, in addition to its effect on 
MEK1/2-Rac, LeTx also leads to the overactivation of RhoA through the inhibition of another MAPK pathway, leading to the inhibition of cell migration and invasion.

Our data show that LeTx is a potent in vitro targeted toxin that could inhibit astrocytoma cell motility and invasion. This study only paves the way for prospectively thorough studies and opens new horizons to novel therapeutic approaches. Consequently, further studies should be conducted to investigate the underlying mechanism behind the effect of LeTx on astrocytoma cell motility and the specific MAPK pathway downstream from LeTx. Deciphering signaling pathways impacted by LeTx are of great importance. It will also be significant to further dissect the direct relationship between the MAPK pathways and Rho GTPases in these cells.

\section{Acknowledgements}

The ROI_Tracker software was supplied by David Entenberg and John Condeelis as supported by CA100324 and GM064346. The present study was supported by the Natural Science Department at the Lebanese American University.

\section{References}

1. Deorah S, Lynch CF, Sibenaller ZA and Ryken TC: Trends in brain cancer incidence and survival in the United States: Surveillance, Epidemiology, and End Results Program, 1973 to 2001. Neurosurg Focus 20: E1, 2006.

2. von Deimling A, von Ammon K, Schoenfeld D, Wiestler OD, Seizinger BR and Louis DN: Subsets of glioblastoma multiforme defined by molecular genetic analysis. Brain Pathol 3: 19-26, 1993.

3. DeAngelis LM: Brain tumors. N Engl J Med 344: 114-123, 2001.

4. Moon SY and Zheng Y: Rho GTPase-activating proteins in cell regulation. Trends Cell Biol 13: 13-22, 2003.

5. Yamazaki D, Kurisu S and Takenawa T: Regulation of cancer cell motility through actin reorganization. Cancer Sci 96: 379-386, 2005.

6. El-Sibai M, Pertz O, Pang H, Yip SC, Lorenz M, Symons M, Condeelis JS, Hahn KM and Backer JM: RhoA/ROCK-mediated switching between Cdc42- and Rac1-dependent protrusion in MTLn3 carcinoma cells. Exp Cell Res 314: 1540-1552, 2008.

7. Ananthakrishnan R and Ehrlicher A: The forces behind cell movement. Int J Biol Sci 3: 303-317, 2007.

8. Vega FM, Fruhwirth G, Ng T and Ridley AJ: RhoA and RhoC have distinct roles in migration and invasion by acting through different targets. J Cell Biol 193: 655-665, 2011.

9. El-Sibai $\mathrm{M}$ and Backer JM: Phospholipase $\mathrm{C} \gamma$ negatively regulates $\mathrm{Rac} / \mathrm{Cdc} 42$ activation in antigen-stimulated mast cells. Eur J Immunol 37: 261-270, 2007.

10. Moorman JP, Luu D, Wickham J, Bobak DA and Hahn CS: A balance of signaling by Rho family small GTPases RhoA, Rac1 and $\mathrm{Cdc} 42$ coordinates cytoskeletal morphology but not cell survival. Oncogene 18: 47-57, 1999.

11. Ridley AJ: RhoA, RhoB and RhoC have different roles in cancer cell migration. J Microsc 251: 242-249, 2013.

12. Buchsbaum RJ: Rho activation at a glance. J Cell Sci 120 : $1149-1152,2007$

13. O'Connor K and Chen M: Dynamic functions of RhoA in tumor cell migration and invasion. Small GTPases 4: 141-147, 2013.

14. Ridley AJ: Life at the leading edge. Cell 145: 1012-1022, 2011.

15. Ellenbroek SI and Collard JG: Rho GTPases: Functions and association with cancer. Clin Exp Metastasis 24: 657-672, 2007.

16. Vega FM and Ridley AJ: Rho GTPases in cancer cell biology. FEBS Lett 582: 2093-2101, 2008.

17. Boettner B and Van Aelst L: The role of Rho GTPases in disease development. Gene 286: 155-174, 2002.
18. Khalil BD, Hanna S, Saykali BA, El-Sitt S, Nasrallah A, Marston D, El-Sabban M, Hahn KM, Symons M and El-Sibai M: The regulation of RhoA at focal adhesions by StarD13 is important for astrocytoma cell motility. Exp Cell Res 321: 109-122, 2014.

19. Lauffenburger DA and Horwitz AF: Cell migration: A physically integrated molecular process. Cell 84: 359-369, 1996.

20. Bermudez O, Pagès G and Gimond C: The dual-specificity MAP kinase phosphatases: Critical roles in development and cancer. Am J Physiol Cell Physiol 299: C189-C202, 2010.

21. Vial E, Sahai E and Marshall CJ: ERK-MAPK signaling coordinately regulates activity of Rac1 and RhoA for tumor cell motility. Cancer Cell 4: 67-79, 2003.

22. Sebolt-Leopold JS, Dudley DT, Herrera R, Van Becelaere K, Wiland A, Gowan RC, Tecle H, Barrett SD, Bridges A, Przybranowski S, et al: Blockade of the MAP kinase pathway suppresses growth of colon tumors in vivo. Nat Med 5: 810-816, 1999.

23. Krueger JS, Keshamouni VG, Atanaskova N and Reddy KB: Temporal and quantitative regulation of mitogen-activated protein kinase (MAPK) modulates cell motility and invasion. Oncogene 20: 4209-4218, 2001.

24. Zohrabian VM, Forzani B, Chau Z, Murali R and JhanwarUniyal M: Rho/ROCK and MAPK signaling pathways are involved in glioblastoma cell migration and proliferation. Anticancer Res 29: 119-123, 2009.

25. Bradley KA, Mogridge J, Mourez M, Collier RJ and Young JA: Identification of the cellular receptor for anthrax toxin. Nature 414: 225-229, 2001.

26. Scobie HM, Rainey GJ, Bradley KA and Young JA: Human capillary morphogenesis protein 2 functions as an anthrax toxin receptor. Proc Natl Acad Sci USA 100: 5170-5174, 2003.

27. Abi-Habib RJ, Urieto JO, Liu S, Leppla SH, Duesbery NS and Frankel AE: BRAF status and mitogen-activated protein/ extracellular signal-regulated kinase kinase $1 / 2$ activity indicate sensitivity of melanoma cells to anthrax lethal toxin. Mol Cancer Ther 4: 1303-1310, 2005.

28. Abrami L, Liu S, Cosson P, Leppla SH and van der Goot FG: Anthrax toxin triggers endocytosis of its receptor via a lipid raftmediated clathrin-dependent process. J Cell Biol 160: 321-328, 2003.

29. Melnyk RA and Collier RJ: A loop network within the anthrax toxin pore positions the phenylalanine clamp in an active conformation. Proc Natl Acad Sci USA 103: 9802-9807, 2006.

30. Huang D, Ding Y, Luo WM, Bender S, Qian CN, Kort E, Zhang ZF, VandenBeldt K, Duesbery NS, Resau JH, et al: Inhibition of MAPK kinase signaling pathways suppressed renal cell carcinoma growth and angiogenesis in vivo. Cancer Res 68: 81-88, 2008.

31. During RL, Li W, Hao B, Koenig JM, Stephens DS, Quinn CP and Southwick FS: Anthrax lethal toxin paralyzes neutrophil actin-based motility. J Infect Dis 192: 837-845, 2005.

32. Dudley DT, Pang L, Decker SJ, Bridges AJ and Saltiel AR: A synthetic inhibitor of the mitogen-activated protein kinase cascade. Proc Natl Acad Sci USA 92: 7686-7689, 1995.

33. Duesbery NS, Webb CP, Leppla SH, Gordon VM, Klimpel KR, Copeland TD, Ahn NG, Oskarsson MK, Fukasawa K, Paull KD, et al: Proteolytic inactivation of MAP-kinase-kinase by anthrax lethal factor. Science 280: 734-737, 1998.

34. Abi-Habib RJ, Singh R, Leppla SH, Greene JJ, Ding Y, Berghuis B, Duesbery NS and Frankel AE: Systemic anthrax lethal toxin therapy produces regressions of subcutaneous human melanoma tumors in athymic nude mice. Clin Cancer Res 12: 7437-7443, 2006.

35. Chopra AP, Boone SA, Liang X and Duesbery NS: Anthrax lethal factor proteolysis and inactivation of MAPK kinase. J Biol Chem 278: 9402-9406, 2003.

36. Ha SD, Ng D, Pelech SL and Kim SO: Critical role of the phosphatidylinositol 3-kinase/Akt/glycogen synthase kinase-3 signaling pathway in recovery from anthrax lethal toxin-induced cell cycle arrest and MEK cleavage in macrophages. J Biol Chem 282: 36230-36239, 2007.

37. Hanna S and El-Sibai M: Signaling networks of Rho GTPases in cell motility. Cell Signal 25: 1955-1961, 2013. 